\title{
Current trends in single cell analysis
}

\author{
Petra Dittrich • Norbert Jakubowski
}

Published online: 27 September 2014

(C) Springer-Verlag Berlin Heidelberg 2014

Single cell analysis is a rapidly emerging research field that fascinates researchers from various disciplines: biologists, because it may reveal insights into fundamental aspects like evolution, cell adaptation, and cell differentiation; medical doctors and pharmacists, because it may have an impact in the understanding of diseases such as cancer, the functioning of the immune system, or the emergence of antibiotic resistances; biotechnologists, because it may further advance evolutionary biotechnology; analytical chemists, because it forces any analytical method to its limits. Single cells provide an extremely difficult matrix for analysis as they are small in size (approximately $1-50 \mu \mathrm{m}$ ) and volume (1 fL for a bacterial cell!) and they consist of many different types of biomolecules with concentrations in a very large dynamic range (few copies of a molecule next to concentrations of $\mathrm{mM}$ ). Moreover, a living cell is not a stable "mixture," it grows and, thereby, exhibits different cell cycle stages, it has a metabolism, and it may respond to slight variations in the external chemical, physical, or mechanical environment. For these reasons, suspensions of cells are used in classic analytical approaches to analyze cellular content. However, such types of bulk measurements provide average values of cell populations but obscure individual behavior of the cells.

Published in the topical collection Single Cell Analysis with guest editors Petra Dittrich and Norbert Jakubowski.

\section{P. Dittrich}

ETH Zurich, Vladimir-Prelog-Weg 3, HCI E 313, 8093 Zurich, Switzerland

e-mail: dittrich@org.chem.ethz.ch

\section{N. Jakubowski ( $\bowtie)$}

BAM Bundesanstalt für Materialforschung und -prüfung,

Fachbereich 1.1, "Anorganische Spurenanalytik",

Richard-Willstätter-Straße 11, 12489 Berlin, Germany

e-mail: norbert.jakubowski@bam.de
Even isogenic cells (derived from the same parent cell) can differ significantly because each single cell is embedded in a local microenvironment, interacts with neighboring cells, or can develop in a stochastic way differently from others (intrinsic noise). These heterogeneities can be observed on all levels of biomolecules such as the transcriptome, proteome, and the metabolome level. However, every type of molecule requires again a different analytical method. Current analytical methods for single cell analysis are either based on non-nvasive, mainly optical methods ("biological" analysis) or on the chemical analysis of lysate derived from individual cells. Noninvasive methods allow the observation of living cells, also over longer time scales, and have been routinely performed by means of fluorescence microscopy and related techniques. They address typically only one or very few target molecules, but are highly specific and sensitive. More recent approaches use label-free analytical methods, e.g., Raman spectroscopy and impedance measurements, with the big advantage that tedious labeling strategies are not required any more and cells can be observed without any intervention in their nature. On the other hand, the chemical analysis of cell lysates allows detailed quantification of target molecules, e.g., by mass spectrometry [1] or immunoassays [2]. Beyond the tremendous increase of the sensitivity of many analytical instruments in recent years, the advances in cell and fluid handling attributable to microfluidics technology has pushed the field of single-cell analysis to yet another level. Microfluidics provides solutions to capture and/or encapsulate single cells in traps or droplets, and to control the environment at an unprecedented accuracy so that reliable studies of individual cell response become feasible. In addition, aliquoting of cell lysates on microfluidic platforms is within reach. Microfluidic platforms routinely made of transparent materials such as polymers or glass are very well suited for microscopy and related techniques. In recent years, 
new interfaces have been developed to expand the analytical readout to the standard chemical methods, in particular mass spectrometry (e.g., Küster et al. [3], Verboket et al. [4]).

At this point, we want to emphasize the many comprehensive review articles that discuss the challenges and current approaches of single cell analysis (e.g., Zenobi [5] Fritzsch et al. [6], Schmid et al. [7], Borland et al. [8]). Furthermore, in a recent issue of ABC (406/14) entitled "Multiplex platforms in diagnostics and bioanalytics," the state of the art of those methods is discussed, which are already fit for purpose of measuring at cellular levels. In all these papers "well" established analytical methods are discussed and are frequently applied. The focus of our special issue is the more "hidden" spectroscopies, better to say sleeping beauties, which are just emerging into these new research directions, and their possible combination with microfluidic techniques for cell and fluid handling. The approaches can be divided into label-free methods and methods where target molecules are labeled.

\section{Label-free analysis of single cells}

Label-free methods provide the advantage that the target molecules are not modified and, hence, all processes are supposed to be undisturbed and the cells do not already show a stress reaction attributable to the label. Moreover, label-free measurements can be used to monitor cell size or shape, and to validate the results from achieved label-based methods.

In a model investigation, tip-enhanced Raman scattering (TERS) was combined by Watkins-Mariani et al. (in this topical collection) with atomic force microscopy (AFM) and used for the first time to achieve simultaneous topographical and label-free structural characterization of individual cells to study wound healing just in a single experiment on the nanometer scale. In their study, lipid fluctuations in the plasma membrane of epidermal keratinocytes were studied label-free at multiple time points post-wounding to better understand caveolar bulb distributions and fluctuations during the healing process. In comparison to histologic staining, the authors could demonstrate that only Raman spectroscopy provided the local resolution and sensitivity to achieve information needed to differentiate time-resolved plasma membrane lipid fluctuations. For validation of AFM and spectroscopic results, real time reverse transcription quantitative polymerase chain reaction (RT-qPCR) and immunofluorescence were applied, giving additional informations about the transcript and protein levels.

Due to their special chemical and physical properties, engineered nanoparticles are already present in many consumer products, and a number of articles describing the state of the art methods used to analytically characterize these materials have been recently compiled in a special issue of $\mathrm{ABC}$ (406/ 16, 'Characterization of Nanomaterials in Biological
Samples'). These materials can be set free directly into the environment or can even be incorporated intentionally by different pathways. Thus, analytical methods are needed to study the uptake of nanoparticles by biological systems and, in particular, by cells.

Gold nanoparticles are investigated in the work of Büchner et al. (in this topical collection) in fibroblast cells in a series of pulse-chase experiments with a $30 \mathrm{~min}$ incubation pulse and chase times ranging from $15 \mathrm{~min}$ to $48 \mathrm{~h}$ to study the uptake and transport of nanoparticles in the cell system. The gold nanoparticles and their aggregates are investigated inside the cellular ultrastructure using laser ablation ICP-MS micromapping and surface-enhanced Raman scattering (SERS). Although the nanoparticle is the analytical target in this study, nano-aggregates also serve as SERS nano-probe to generate the signal, and are used to report on the local microenvironment on the surface of the particles. Surprisingly, the sensitivity and the local resolution of both methods are already sufficient to get signals from single cells and nano-particle agglomerates. In this way, information about their localization at the micron scale and their molecular nano-environment, respectively, is obtained and can be related. Thus, the nanoparticle pathway from endocytotic uptake, intracellular processing, to cell division can be followed using both complementing techniques. The results were validated by use of transmission electron microscopy (TEM). The SERS spectra indicate that mainly proteins interact with the gold nanoparticle surface and some functional groups observed persist even throughout the endosomal maturation process.

It is already well known that ICP-MS belongs to the most sensitive methods in atomic spectroscopy, but it is still a challenge to reach absolute limits of detection at the subfemtogram level needed to measure label-free essential elements, in particular metals, directly in a single cell. However, two approaches are discussed in the trends article of Mueller et al. (in this topical collection) where it was possible to detect already metals in single cells by ICP-MS. The first example is the direct injection of intact cells by use of a micro-droplet generator. Here, cells are embedded in a single droplet, which is shot into the plasma directly. With this sample introduction method, metals in yeast cells have been detected already by Shigeta et al. [9] at attogram levels. A similar approach was presented by Verboket et al. [4], but they used a novel microfluidic system for droplet generation and direct injection into the ICP-MS. The core component of the system is based on liquid assisted droplet ejection (LADE) chip, which generates droplets in size ranges from 40 to $60 \mu \mathrm{m}$, in which single cells can be embedded in a highly volatile oil phase. Intensities for ${ }^{56} \mathrm{Fe}^{+}$of about $400 \mathrm{cps}$ have been measured representing $5 \times 10^{8}$ atoms in a single blood cell, a value that agrees well with the bulk concentration measured. The microfluidic chip can be made and reproduced at low costs, and future modifications of the chip design can allow the 
integration of various processes, such as on-chip sample pretreatment and parallel introduction of different sample.

The second example also discussed in the article by Mueller et al. (in this topical collection) was mentioned previously, where laser ablation ICP-MS was applied for micromapping of nanoparticles in single cells. In this review, the authors describe a so-called "differential scanning mode" for laser ablation for the first time, which provides a local resolution much smaller than the laser diameter so that many hundred pixels can be measured per single cell. Additionally, this method can be calibrated by matrix matched standards (more details in the trends article) so that finally the number of nanoparticles up-take by single cells can be calculated. So far, this work is limited by the lateral resolution achieved in the differential scanning mode, which is dominated by the washout of the laser ablation cell, but recently a novel tubelike laser ablation cell in combination with an excimer laser was developed by Wang et al. [10] with fast washout times of $30 \mathrm{~ms}$ (compared with roughly $1 \mathrm{~s}$ in commercial devices) for high spatial resolution, which is a breakthrough in this field. All these developments open the door for a new method: the quantitative elemental microscope with cellular resolution!

Label-free assessment of cell parameters of living cells is demonstrated by the work of Zhu et al. (in this topical collection). In this work, electrical impedance spectroscopy (EIS) is employed to monitor the growth of yeast. To facilitate the immobilization of individual yeast cells, a microfluidic device is used that consists of cell capture sides, where cells can be gently sucked and stably immobilized. The combination with local multifrequency EIS measurements was first successfully validated by the use of monodisperse particles, which showed the high sensitivity and precision of the microfluidic single cell EIS device. When this method is applied to yeast cells, it becomes feasible to discriminate between budding yeast and yeast without a small daughter cell (unbudded yeast) and their orientations in the cell trap. Motion and growth vectors can be determined, (i.e., important parameters of cellular dynamics). For these measurements, a planar and transparent microchip is used; hence, it could be straightforwardly combined with many other analytical spectroscopic techniques and microscopy, and the results could be directly correlated with cell proliferation or cell motility.

\section{Analysis of single cells by use of labeling techniques}

Methods that involve labels usually require additional steps for labeling the target analyte(s), but provide the great advantage of high specificity and sensitivity. The most common label is a fluorescent molecule used to visualize the analyte. Alternatively, fluorescent molecules can serve as reporter to indicate the presence of molecules, including measures of their functionality. This is nicely demonstrated in the study of Dickinson et al. (in this topical collection), where a fluorescent sphingosine reporter was loaded into blood cells to not only prove the presence of the enzyme sphingosine kinase (SK) but also its activity. SK is an enzyme that catalyzes the phosphorylation of sphingosine and therby promotes cell survival, prolifereation, and migration. Overexpression of SK has been associated with a number of cancers, including leukemia, and has been identified as a promising therapeutic target in the treatment of leukemia. To measure SK activity in cells of leukemic origin and purified leukemic blasts, Allbritton and co-workers used an automated system based on single cell capillary electrophoresis and quantified the metabolism of the fluroescent sphingosine reporter [8]. With this system, they could prove that freezing of cells strongly influenced the SK activity and, therefore, fresh cells were critically important for accurate measurements. They measured large heterogeneities in the sample; however, it was unclear if this heterogeneity was also a consequence of the diverse cell types. Therefore, a pure population of leukemic blasts was investigated in more detail. Here, a significant level of heterogeneity was found, but also a lower activity of SK. Intrestingly, the sphingosine reporter was converted into an unidentified compound, obviously by another enzyme, and the authors expressed their hope that this unknown enzyme could be a new drug target in leukemic cells.

By use of stable isotopic labeling of fatty acids with deuterium, the uptake and storage by human mapcrophages of exogenously provided fatty acids have been investigated by Stiebing et al. (in this topical collection). In this study, the authors present the results of time-dependent Raman spectroscopic imaging using a confocal Raman microscope with $0.5 \mu \mathrm{m}$ step size to image single THP-1 macrophages incubated with deuterated arachidonic acid. This polyunsaturated fatty acid is an essential omega-6-fatty acid and plays an important role in the cellular inflammatory signaling as a precursor of icosanoids. The authors show that arachidonic acid is stored in lipid droplets but the storage efficiency in lipid droplets is lower than in cells incubated with deuterated palmitic acid. Results were validated by use of gas chromatography, which provides additional information of other fatty acids in the cell, such as precursors and metabolites. These studies show that Raman microscopy is already a powerful nondestructive and noninvasive tool to spatially resolve and investigate the chemical composition of a single cell. In the conclusions, the authors summarize their experience "Only through combination of several different methods like Raman micro-spectroscopy and gas chromatography will it be possible to fully understand the molecular processes in cells."

In principle X-ray fluorescence microscopy based on synchrotron radiation $(\mathrm{SXRF})$ is a label-free method that provides subcelluar resolution ( $<100 \mathrm{~nm}$ ) and outstanding sensitivities for many elements, and is often applied for elemental imaging and speciation if combined with $\mu \mathrm{X}$-ray absorption 
spectroscopy ( $\mu$ XAS). In the review from Roudeau et al. (in this collection) this powerful technique is combined with fluorescence microscopy after appropriate labeling to correlate the elemental distribution with cell structures, for instance organelles. The review article describes the method used for simultaneous application of fluorescence microscopy to visualize cells organelles without affecting the intrinsic elemental distribution, which is essential for the measurements by SXRF. The difficulties and, in particular, the challenges for cryogenic samples preparation is discussed for both methods, and first applications are presented from different groups.

The state-of-the-art for a high-throughput qualitative and quantitative multiparameter analysis is flow cytometry, as has been reviewed recently by Haselgrübler et al. [11], in a special issue of ABC (406/14, Multiplex Platforms in Diagnostics and Bioanalytics). For this purpose, a cell sample is treated with fluorescence probes (e.g., antibodies), and the cell suspension is formed to a liquid stream, which allows the aligned cells to pass a light beam for fluorescence sensing. Although extremely successful, the technique is limited by spectral overlap (which limits the number of colors to be used simultaneously), fluorescence dye quenching, and auto-fluorescence of the sample. An alternative and novel technique (CyTOF), which is free of these limitations, was developed by Tanner and colleagues [12, $13]$ and is becoming very popular as "mass cytometry" attributable to the pioneering work of Gary Nolan's group [14] (for more details see the article by Mueller et al. (this paper collection). In contrast to the fluorophores used in conventional flow cytometry, in mass cytometry metal labeling of antibodies are used and ICP-time-of-flight-MS is employed for detection. In this article, Mueller discusses the chemistry of the labeling process, the fundamentals of the technology, and very recent applications. Using the CyTOF-ICP-MS instrument, Gunther et al. in Zurich used the tube-like ablation cell approach mentioned before for spatially resolved multiplexing immuno-imaging of 32 proteins and their modifications in formalin-fixed and paraffinembedded breast cancer tissue samples and human mammary epithelial (HMLE) cells (for more details see the article by Mueller et al. [15]. This pioneering work is a huge step forward to the development of highly multiplex- and multiparametric immune-imaging with cellular resolution.

\section{Outlook}

All studies discussed so far clearly demonstrate that various analytical methods can already be used for single-cell analysis and together they offer valuable biologically relevant insights into the composition of the individual cell, as well as the uptake of metallodrugs and engineered nanoparticles. Keeping in mind that a cell is a very complex and small machinery with many life functions, which consist of many millions of proteins and their post-translational modifications and, additionally, many billions of metabolites, we get only a snapshot of a very low number of parameters of a highly dynamic and rather complex system. From the point of view of systems biology, the information that we can measure is by far not sufficient; therefore, complementary methods are required if multiparametric function or malfunction of diseased cells have to be investigated. Thus, the guest editors want to foster the idea of "multimodal spectroscopies" to be applied simultaneously to one and the same cell system to get reliable and complementary results. Only the combination of different analytical techniques can expose the secrets of complex systems for a better understanding of the processes and dynamics of biologically or medically relevant cells. Furthermore, the coupling of these methods with microchip-based systems is essential for sorting, fixing, separating of cells, or even for controlling the micro-environment or treatment conditions. Finally, to overcome the biological variability, a large number of cells have to be measured simultaneously or in parallel so that development of "high-throughput" methods, together with statistical data treatment, is still of actual interest. To achieve this, new smart methods are required that facilitate multiple (subsequent or simultaneous) measurements on individual cells. For this, new interfacing platforms are needed as well as new strategies for fractionating or aliquoting ultrasmall volumes, thereby avoiding dilutions of cell lysates.

We are convinced that combinations of microfluidic platforms and multimodal spectroscopy open the gate to the nextgeneration single-cell studies with defined stimulation of cells and analysis of cell responses, simultaneously for as many different biomolecules as possible. Synergistic cooperation across disciplines and research groups with complementary expertise are the essential requirements to develop the needed tools for the acquisition of experimental data and, at the same time, to ensure reasonable data analysis and interpretation together with its meaningful correlation to the complex cell machinery.

\section{References}

1. Ibanez A, Fagerer S, Schmidt A, Urban P, Jefimovs K, Geiger P, Heinemann M, Zenobi R (2013) Mass spectrometry-based metabolomics of single yeast cells. Proc Natl Acad Sci U S A 110:87908794

2. Eyer K, Stratz S, Kuhn P, Küster SK, Dittrich PS (2013) Implemening ezyme-linked immunosorbent assays on a microfluidic chip to quantify intracellular molecules in single cells. Anal Chem 85:3280-3287 
3. Küster SK, Fagerer SR, Verboket PE, Eyer K, Jefimovs K, Zenobi R, Dittrich PS (2013) Interfacing droplet microfluidics with matrixassisted laser desorption ionization mass spectrometry: label-free content analysis of single droplets. Anal Chem 85:1285-1289

4. Verboket PE, Borovinskaya O, Meyer N, Günther D, Dittrich PS (2014) A new microfluidics-based droplet dispenser for ICPMS. Anal Chem 86:6012-6018

5. Zenobi R (2014) Single-cell metabolomics: analytical and biological perspectives. Science 342(6163): 1201

6. Fritzsch FS, Dusny C, Frick O, Schmid A (2012) Single-cell analysis in biotechnology, systems biology, and biocatalysis. Annu Rev Chem Biomol Eng 3:129-155

7. Schmid A, Kortmann H, Dittrich PS, Blank LM (2010) Chemical and biological single-cell analysis. Curr Opin Biotechnol 21:12-20

8. Borland LM, Kottegoda S, Phillips KS, Allbritton NL (2008) Chemical analysis of single cells. Annu Rev Anal Chem 1:191-227

9. Shigeta K, Koellensperger G, Rampler E, Traub H, Rottmann L, Panne U, Okino A, Jakubowski N (2013) Sample introduction of single selenized yeast cells (Saccharomyces cerevisiae) by micro droplet generation into ICP-sector field mass spectrometer for labelfree detection of trace elements. J Anal At Spectrom 28:637-645

10. Wang HAO, Grolimund D, Giesen C, Borca CN, Shaw-Stewart JRH, Bodenmiller B, Gunther D (2013) Fast chemical imaging at high spatial resolution by laser ablation inductively coupled plasma mass spectrometry. Anal Chem 85:10107-10116

11. Haselgrübler T, Haider M, Ji B, Juhasz K, Sonnleitner A, Balogi Z, Hesse J (2014) Anal Bioanal Chem 406:3279-3296

12. Tanner SD, Bandura DR, Ornatsky O, Baranov VI, Nitz M, Winnik MA (2008) Flow cytometer with mass spectrometer detection for massively multiplexed single-cell biomarker assay. Pure Appl Chem 80:2627-2641

13. Bandura DR, Baranov VI, Ornatsky OI, Antonov A, Kinach R, Lou XD, Pavlov S, Vorobiev S, Dick JE, Tanner SD (2009) Mass Cytometry: Technique for real time single cell multitarget immunoassay based on inductively coupled plasma time-of-flight mass spectrometry. Anal Chem 81:6813-6822

14. Bendall SC, Simonds EF, Qiu P, Amir EAD, Krutzik PO, Finck R, Bruggner RV, Melamed R, Trejo A, Ornatsky OI, Balderas RS, Plevritis SK, Sachs K, Pe'er D, Tanner SD, Nolan GP (2011) Single-cell mass cytometry of differential immune and drug responses across a human hematopoietic continuum. Science 332(6030):687-696

15. Giesen C, Wang HA, Schapiro D, Zivanovic N, Jacobs A, Hattendorf B, Schüffler PJ, Grolimund D, Buhmann JM, Brandt S (2014) Highly multiplexed imaging of tumor tissues with subcellular resolution by mass cytometry. Nat Methods. doi:10.1038/NMETH.2869

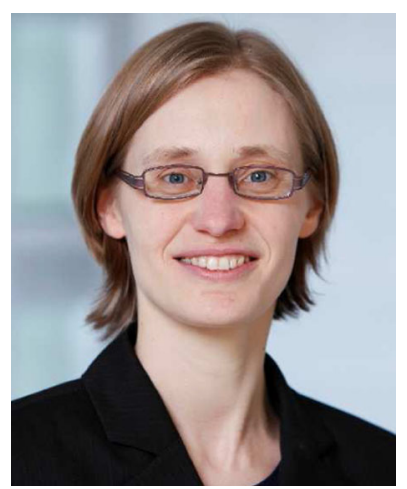

Petra Dittrich has been Assistant Professor for Bioanalytics at ETH Zurich (Switzerland) since 2008. She studied chemistry at Bielefeld University (Germany) and Universidad de Salamanca (Spain), and earned her $\mathrm{PhD}$ at the Max Planck-Institute for Biophysical Chemistry (Göttingen, Germany) in 2003. After another year as a postdoctoral fellow at the MPI Göttingen, she worked at the Institute for Analytical Sciences (Dortmund, Germany) (2004-2008). For research stays, she was at the Cornell University (2002) and the University of Tokyo (2005). Her research focuses on the miniaturization of high-sensitivity devices for chemical and biological analyses, particularly for single-cell analysis, and the development of new methods for microfluidic-aided organization of materials and artificial cells.

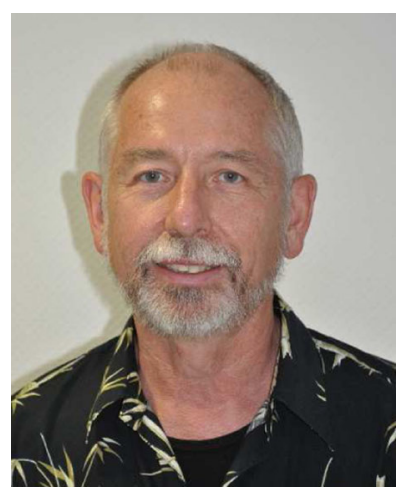

Norbert Jakubowski is presently head of the division "Inorganic Trace Analysis" at the BAM Federal Institute for Materials Research and Testing, Berlin, Germany (since 2009). He studied plasma-physics at the University of Essen/Duisburg and worked at the Institute for Analytical Sciences (Dortmund, Germany) (1982-2009). He received his $\mathrm{PhD}$ at the University of Stuttgart Hohenheim in 1991. His research interests are related to analytical chemistry in general with special interest in the development of instruments, methods, and problemorientated procedures based on the use of plasma sources (inductively coupled plasma, glow discharge) for elemental mass spectrometry of solid and liquid samples. At BAM he recently started a new research direction towards using laser ablation ICP-MS for bio- and immunoimaging of tissues and single cells. 